\title{
Coparentalidade e comportamento da criança no contexto do divórcio: Uma revisão sistemática
}

\author{
Tatiane M. B. Ambros, Beatriz P. Coltro, Mauro Luís Vieira e Fernanda M. Lopes \\ Departamento de Psicologia, Universidade Federal de Santa Catarina (UFSC)
}

Submissão: 21 jan. 2021.

Aceite: 9 ago. 2021.

Notas dos autores

Tatiane M. B. Ambros (D) https://orcid.org/00oo-0002-5387-0590

Beatriz P. Coltro (D) https://orcid.org/00oo-0002-8471-3141

Mauro Luís Vieira (D) https://orcid.org/00oo-0003-0541-4133

Fernanda M. Lopes (D) https://orcid.org/00oo-0002-4853-7670

Financiamento: As coautoras Beatriz P. Coltro e Tatiane M. B. Ambros, autoras do trabalho, são bolsistas da Coordenação de Aperfeiçoamento de Pessoal de Nível Superior (Capes) de mestrado e doutorado na Universidade Federal de Santa Catarina. O coautor Dr. Mauro Luís Vieira é bolsista de produtividade do Conselho Nacional de Desenvolvimento Científico e Tecnológico (CNPq) ( ${ }^{\circ}$ 306811/2019-7).

Correspondências referentes a este artigo devem ser enviadas para Tatiane M. B. Ambros, Universidade Federal de Santa Catarina, Rua Engenheiro Agrônomo Andrei Cristian Ferreira, s/n, Trindade, Florianópolis, SC, Brasil. CEP 88040-900.E-mail: tatianeambros@yahoo.com.br 


\title{
Resumo
}

A qualidade da relação coparental após o divórcio dos pais afeta significativamente a relação pais-fiIhos e o desenvolvimento infantil. O objetivo do estudo foi realizar uma revisão sistemática sobre as relações entre coparentalidade e comportamento da criança em famílias divorciadas. A revisão sistemática foi conduzida de acordo com as recomendações metodológicas do Preferred Reporting Items for Systematic Reviews and Meta-Analyses (PRISMA). As buscas foram realizadas em seis bases de dados, nacionais e internacionais, com os termos coparentalidade, divórcio e comportamento da criança, e termos correlatos, em português, inglês e espanhol. Foram incluídos 11 artigos publicados entre 2010 e junho de 2020. Por meio da análise dos resultados desses artigos, constatou-se que o apoio coparental após o divórcio favorece desfechos positivos no comportamento infantil. Contudo, a existência de conflito coparental representa fator de risco para problemas de comportamento na criança, inclusive em famílias em que há apoio e comunicação coparental.

Palavras-chave: coparentalidade, crianças, divórcio, revisão sistemática, relações familiares

\section{COPARENTING AND CHILD BEHAVIOR IN THE CONTEXT OF DIVORCE: A SYSTEMATIC REVIEW}

\begin{abstract}
The quality of the coparental relationship after parental divorce significantly affects the parents-children relationship and the child development. The objective of the study was to conduct a systematic review on the relationship between coparenting and child behavior in divorced families. The systematic review was conducted according to the methodological recommendations of the Preferred Reporting Items for Systematic Reviews and Meta-Analyses (PRISMA). The searches were carried out in six national and international databases, using the words coparenting, divorce and child behavior, and similar terms, in Portuguese, English and Spanish. Eleven articles published between 2010 and June 2020 were included. The main results of the studies showed that coparental support after divorce favors more positive outcomes in child behavior. On the other hand, the existence of coparental conflict represents risk factor for child behavioral problems, including in families whit coparental support and communication exist.
\end{abstract}

Keywords: coparenting, children, divorce, systematic review, family relations

\section{COPARENTALIDAD Y COMPORTAMIENTO INFANTIL EN EL CONTEXTO DEL DIVORCIO: UNA REVISIÓN SISTEMÁTICA}

\section{Resumen}

La calidad de la relación coparental después del divorcio de los padres afecta significativamente la relación entre padres e hijos y el desarrollo infantil. El objetivo del estudio fue realizar una revisión sistemática sobre la relación entre coparentalidad y comportamiento del niño en familias divorciadas. La revisión sistemática se realizó conforme las recomendaciones metodológicas del Preferred Reporting 
Items for Systematic Reviews and Meta-Analyses (PRISMA). Las búsquedas se realizaron en seis bases de datos, nacionales e internacionales, con los términos coparentalidad, divorcio y comportamiento infantil en portugués, inglés y español. Se incluyeron 11 artículos publicados entre 2010 y junio de 2020. Los principales resultados de los estudios mostraron que el apoyo coparental después del divorcio favorece resultados más positivos en el comportamiento infantil. Sin embargo, la existencia de conflicto coparental representa factor de riesgo para problemas de comportamiento en niños, incluso en familias que existe el apoyo y la comunicación coparental.

Palabras clave: coparentalidad, niños, divorcio, revisión sistemática, relaciones familiares 
A família é uma instituição social dinâmica e multifacetada, integrada por aqueles que compartilham uma história e projetos de vida em comum. Considerada um dos principais contextos de desenvolvimento de seus integrantes, particularmente na infância, a família é composta por uma série de relações emocionais complexas e interdependentes de seus integrantes que são influenciadas por fatores macrossociais - como cultura, história e aspectos socioeconômicos (McGoldrick \& Shibusawa, 2016). Dessa maneira, diversas mudanças históricas afetam a estrutura e as funções da família, sendo a dissolução conjugal e/ou o divórcio um dos eventos familiares em maior crescimento no século XXI na sociedade ocidental (Greene et al., 2016; Raley \& Sweeney, 2020).

De acordo com dados do Instituto Brasileiro de Geografia e Estatística (2019), os pedidos de divórcio têm aumentado a cada ano; em 2018 foi registrado um divórcio para cada três registros de casamento civil no país. A separação conjugal é um evento de transição familiar responsável por inúmeras mudanças potencialmente estressantes, uma vez que provoca desequilíbrios e reorganizações nos papéis e funções dos membros da família, especialmente quando o casal tem filhos. Nesse contexto, pode-se afirmar que a experiência do divórcio dos pais durante a infância se configura como um fator de risco para o desenvolvimento dos filhos, cujo impacto será definido de acordo com os fatores de proteção da criança e as condições e habilidades dos pais para lidar com essa transição (Greene et al., 2016; Lamela \& Figueiredo, 2016; Sands et al., 2017).

A separação dos pais não dissolve o sistema familiar como um todo, mas tão somente sua relação conjugal, sendo necessário reorganizar a dinâmica e a comunicação para que as funções parentais continuem sendo desempenhadas. Enquanto o exercício da parentalidade se define pela relação da criança com cada um de seus pais, individualmente, e pelas atividades e pelos comportamentos de cuidado dos pais com seus filhos, a coparentalidade se refere ao modo como dois ou mais adultos compartilham essas atribuições no interjogo de papéis que se relacionam com o cuidado global da criança, envolvendo responsabilidade conjunta pelo bem-estar dela (Feinberg, 2003; McHale et al., 2004). Dada essa definição, a coparentalidade existe independentemente da estrutura familiar, desde que duas ou mais pessoas se corresponsabilizem pelos cuidados para/com a criança.

Feinberg (2003) propôs um modelo teórico para explicar a estrutura da coparentalidade por meio de uma visão ecológica e multicomponente, oferecendo uma compreensão sobre como cada componente influencia no ajustamento dos pais, na parentalidade, na relação interparental e no comportamento da criança. De acordo com o autor, a coparentalidade inclui quatro dimensões, a saber: 1. concordância-discordância entre a dupla parental acerca das práticas parentais; 2 . divisão de tarefas (domésticas, cuidados com a criança e financeiras); 3. gerenciamento conjunto das interações familiares; e 4. suporte-sabotagem ao papel parental do parceiro. 
Concordância-discordância entre a dupla parental é a dimensão da coparentalidade associada ao grau de entendimento entre a díade parental em assuntos relacionados com a criança, tais como princípios morais, disciplina, formas de prestação de cuidados, decisões sobre a educação ou necessidades emocionais das crianças. A dimensão divisão de tarefas corresponde à partilha entre a díade coparental das obrigações das rotinas diárias de cuidados à criança, bem como à divisão das responsabilidades dos assuntos financeiros, médicos e legais relacionados com as crianças. Esse componente da coparentalidade tem um expressivo impacto na satisfação com a relação coparental e com os níveis de estresse parental, em que quanto maior for a divisão de tarefas, menor será o estresse no desempenho das funções parentais e maior será satisfação com a relação com o outro (Feinberg, 2003).

A dimensão gerenciamento conjunto das interações familiares possui foco na interação entre os responsáveis, a qualidade do funcionamento estrutural da família dependente do componente da coparentalidade. Este pode ser entendido englobando três aspectos: 1. gestão por parte de pais e mães sobre os próprios comportamentos e sobre a comunicação com os outros dentro do sistema familiar; 2 . a gestão das fronteiras que são estabelecidas pelos comportamentos e pelas atitudes parentais, que contribuem para o engajamento ou a exclusão de outros membros da família; 3. relações familiares equilibradas, ou seja, o modo como um dos membros da díade parental assume a liderança, enquanto o outro em determinada situação se retrai. A dimensão suporte-sabotagem consiste na qualidade e no grau do suporte recíproco entre a díade. Expressões de afeto positivo, reforço, apoio emocional e respeito perante a autoridade e contributos do outro membro do par coparental são manifestações do suporte esperado entre os pais (Feinberg, 2003).

As mesmas dimensões propostas por Feinberg (2003) encontram correlatos e sobreposições nas propostas teórico-metodológicas apresentadas por Margolin et al. (2001). Esses autores elaboraram um questionário que medeia três dimensões coparentais, cooperação e conflito e triangulação. Ademais, de acordo com a revisão metanalítica de Teubert e Pinquart (2010), o modelo de Feinberg (2003) contempla suficientemente bem os elementos componentes pelos quais a coparentalidade é investigada em estudos empíricos, nomeadamente a cooperação, a concordância, o acordo no cuidado e na educação da criança, o conflito e a triangulação. Uma recente revisão de literatura sobre instrumentos de avaliação da coparentalidade sustenta que suas dimensões tendem a se localizar em dois grupos distintos: aqueles relacionados ao apoio e à cooperação (incluindo comunicação, respeito, trabalho em equipe, aliança, confiança, entre outros) e outros relacionados ao conflito coparental (como triangulação, desentendimento, sabotagem, hostilidade, coparentalidade restritiva, entre outros), com análises fatoriais indicando soluções de uma a quatro dimensões para o fenômeno (Cusí et al., 2020). 
Apesar da heterogeneidade nas definições e conceptualizações de modelos sobre a coparentalidade, evidências têm apoiado a hipótese de que a coparentalidade negativa (também denominada competitiva) representa um fator de risco para o desenvolvimento de psicopatologias na infância, enquanto a coparentalidade cooperativa é considerada um fator de proteção aos filhos (Cusí et al., 2020; Teubert \& Pinquart, 2010). Nesse sentido, a coparentalidade se torna um dos principais elementos do contexto familiar com acentuada relevância para o desenvolvimento das crianças, independentemente da estrutura familiar (Solmeyer et al., 2014; Zemp et al., 2018).

Quando os pais se divorciam, a coparentalidade pode representar o único sistema de relações mantido pelo casal (Margolin et al., 2001). Há evidências de que a manutenção de uma relação amigável e com comunicação suficiente entre os pais após o divórcio está relacionada a melhores desfechos tanto para a criança quanto para os pais (Herrero et al., 2020). Contudo, uma recente revisão de literatura identificou resultados inconsistentes a respeito dos efeitos das mudanças na estrutura familiar (como o divórcio) sobre o desenvolvimento dos filhos, evidenciando que outros fatores, como o estresse, os impactos socioeconômicos e as características da criança, podem contribuir para a explicação desses efeitos no contexto da dissolução conjugal dos pais (Hadfield et al., 2018).

A revisão sistemática realizada por Lamela e Figueiredo (2016), que contou com estudos publicados entre os anos de 2000 e 2014 sobre os efeitos da coparentalidade no pós-divórcio sobre o comportamento da criança, demonstrou que o conflito coparental está associado ao aumento de sintomas psicopatológicos e problemas comportamentais nos filhos. Por sua vez, estudos comparativos de famílias casadas e divorciadas permitiram a observação de que os efeitos da coparentalidade negativa ou conflituosa podem ser vivenciados pelas crianças independentemente da estrutura familiar, destacando o divórcio como somente mais um contexto em que conflitos coparentais possam surgir.

Diante das contínuas atualizações científicas a respeito do tema, torna-se relevante revisar a literatura recente com o intuito de identificar como a coparentalidade se relaciona ao comportamento da criança após a separação/divórcio dos pais, incluindo seu desempenho acadêmico, bem-estar e comportamentos externalizantes (relacionados à impulsividade, à agressividade e à hiperatividade) e internalizantes (relacionados ao isolamento, ao retraimento e à expressão emocional de tristeza, depressão e/ou ansiedade) (Bolsoni-Silva et al., 2016). Para tanto, esta revisão sistemática de literatura foi realizada com o objetivo de analisar as relações entre coparentalidade e o comportamento da criança em famílias divorciadas. Considerando a multidimensionalidade da coparentalidade (Feinberg, 2003), a presente revisão amplia os resultados já encontrados até o momento ao analisar relações específicas entre as dimensões da coparentalidade e o comportamento da criança em famílias divorciadas, além de fornecer uma análise detalhada sobre possíveis vieses metodológicos encontrados nos artigos retidos. 


\section{Método}

Trata-se de uma revisão sistemática conduzida com base na pergunta de pesquisa: "Quais são as relações entre a coparentalidade e o comportamento da criança em famílias divorciadas?". Os componentes da pergunta seguiram a sigla PECOS: População, Exposição, Comparação, (Outcome) Resultado e (Study) Estudo. Utilizaram-se as diretrizes de descrição para revisão do guia Preferred Reporting Items for Systematic Reviews and Meta-Analyses - PRISMA (Moher et al., 2009), porém sem registro prévio do protocolo (o protocolo utilizado pode ser disponibilizado pelo autor correspondente).

\section{Critérios de busca e inclusão do material na revisão}

A busca sistemática foi realizada em junho de 2020, incluindo artigos empíricos publicados entre 2010 e 2020 (até o mês de junho), sem restrições de idiomas, nas bases de dados PubMed, Web of Science, Psyclnfo, Scopus e SciELO, e na fonte de pesquisa Biblioteca Virtual em Saúde (BVS). A estratégia de busca completa, incluindo os descritores e operadores booleanos utilizados, foi: (coparenting) AND (divorce OR divorcing OR divorced OR marital dissolution OR separation) AND (child behavior OR child mental health OR child adjustment) e seus respectivos termos em português e espanhol.

Como critério de inclusão, consideraram-se estudos primários (empíricos) que tratassem como temática central a relação entre coparentalidade e comportamento da criança no contexto do divórcio/separação. Os critérios de exclusão foram: 1. estudos que não incluíram instrumentos de medida de coparentalidade ou de comportamento da criança; 2. estudos cuja variável da criança foi utilizada como preditora e não como desfecho ou em associação à coparentalidade; 3. artigos duplicados ou não científicos (não indexados em bases de dados); 4. revisões de literatura, metanálises, editoriais, cartas, livros ou capítulos de livro, teses, dissertações, relatos de caso, relatos de experiência e trabalhos apresentados em congresso.

\section{Procedimentos de coleta dos dados documentais}

Após a etapa de busca, duas juízas independentes (para minimizar o viés de seleção) fizeram a seleção dos estudos a partir da leitura dos resumos, com base nos critérios de elegibilidade, por meio do software de uso livre Rayyan ${ }^{\circledR}$ (Ouzzani et al., 2016). Houve a participação de uma terceira juíza para discutir as dúvidas e deliberar, em conjunto, sobre a concordância do processo. Em alguns casos, foi necessária leitura do texto completo para definir se o estudo seria incluído ou excluído. O fluxograma das etapas de busca e seleção dos artigos está apresentado na Figura 1. 
Figura 1

Fluxograma das etapas de busca e seleção dos artigos
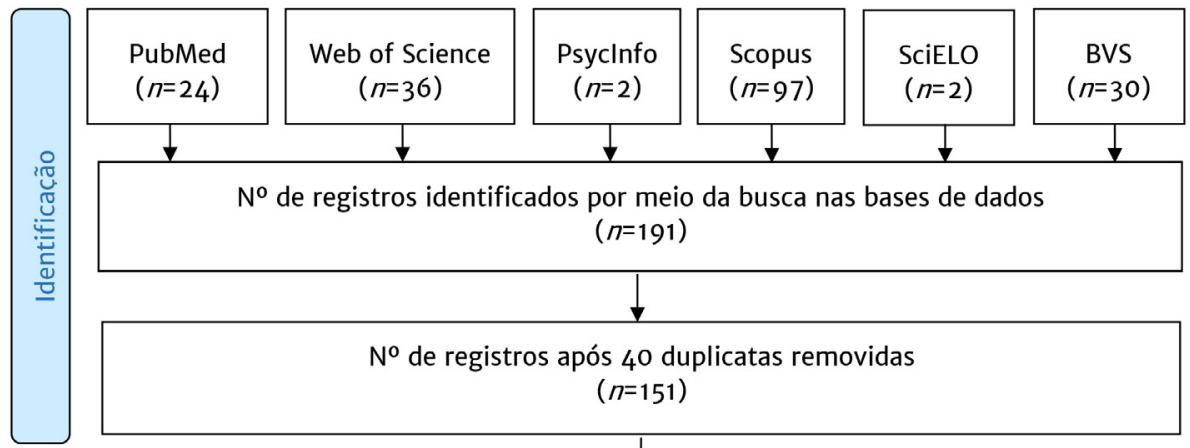

$\mathrm{N}^{0}$ de registros identificados por meio da busca nas bases de dados $(n=191)$
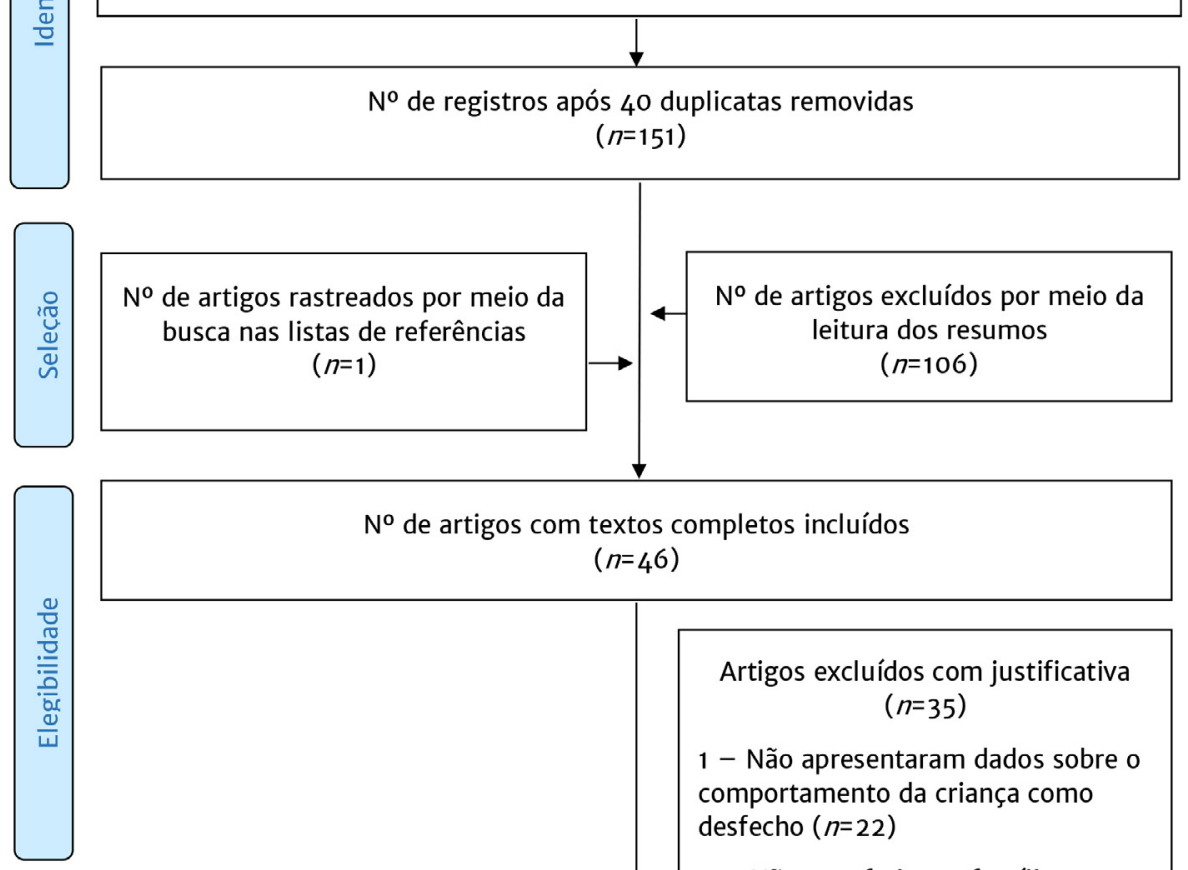

$\mathrm{N}^{\circ}$ de artigos com textos completos incluídos $(n=46)$

Artigos excluídos com justificativa $(n=35)$

1 - Não apresentaram dados sobre o comportamento da criança como desfecho $(n=22)$

2 - Não se referiam a famílias divorciadas ou separadas $(n=13)$

Artigos incluídos para análise final $(n=11)$ 


\section{Procedimentos de extração e análise de dados}

A extração dos dados ocorreu com o auxílio do software Excel e foi realizada, de forma independente, pelas duas juízas que selecionaram os estudos. A terceira juíza fez a revisão da extração dos dados conferindo com a tabela padronizada a partir das características de publicação dos estudos incluídos (objetivos, delineamento, participantes, instrumentos, procedimentos de coleta e análise de dados), incluindo os desfechos relacionados ao comportamento das crianças e as recomendações e limitações descritas nos estudos.

A análise da qualidade metodológica dos artigos foi realizada por meio da aplicação de dois checklists do Joanna Briggs Institute - JBI (2020). O JBI Checklist for Analytical Cross Sectional Studies (contém oito itens) foi utilizado para avaliar os dez artigos correlacionais, após adaptação dos itens 5 e 6 para melhor identificação de vieses nos estudos, considerando seu delineamento. Dessa forma, utilizaram-se esses itens para avaliar a identificação ou não de variáveis intervenientes ou variáveis controle (item 5) e se estratégias adequadas foram descritas e aplicadas para lidar com tais variáveis (item 6). O JBI Checklist for Quasi-experimental Studies (contém nove itens) foi aplicado ao único artigo quase experimental recuperado, sem adaptações.

\section{Resultados}

\section{Caracterização dos estudos}

Esta revisão encontrou estudos oriundos apenas de seis países: Estados Unidos $(n=5)$, Espanha $(n=2)$, Portugal $(n=1)$, Índia $(n=1)$, China $(n=1)$ e Austrália $(n=1)$. Em relação às amostras, os estudos foram conduzidos com famílias, incluindo crianças e/ou seus respectivos pais e mães separados ou divorciados. A maioria dos estudos $(n=7)$ contou majoritariamente com mães como participantes (de 61\% a 100\%). Por sua vez, Amato et al. (2011) não indicaram o gênero do respondente. Nesse aspecto, houve duas exceções: Dyer et al. (2018) investigaram somente a percepção paterna, e o estudo de Jiménez-García et al. (2019) derivou seus dados de documentos judiciais. No estudo de Baxter et al. (2011), os professores também forneceram informações sobre a criança, e, em dois artigos (Baxter et al., 2011; Karberg \& Cabrera, 2020), pais casados também foram incluídos, além dos separados. A investigação conduzida por Jiménez-García et al. (2019) utilizou como fonte de dados arquivos de processos judiciais de divórcio, acessando as informações indiretamente.

A maioria dos estudos fundamentou a investigação a partir da compreensão dos efeitos do divórcio sobre o comportamento da criança, e oito artigos também incluíram definições de coparentalidade a priori, defendendo o fenômeno como uma relação ou interação conjunta entre os pais com o objetivo de coordenar os cuidados com os filhos. Houve consenso de que a coparentalidade pode ser positiva ou negativa a depender da qualidade da cooperação, da comunicação e do grau de conflito existente entre os pais. 
A pesquisa de Dyer et al. (2018) incluiu o desenvolvimento e a validação de uma escala de coparentalidade para pais (homens) não residentes com os filhos, e seis artigos não utilizaram medidas validadas para avaliar a coparentalidade dos participantes, referindo o uso de perguntas específicas sobre frequência de contato e/ou existência de cooperação ou conflito entre os pais. As principais dimensões da coparentalidade medidas pelos estudos foram apoio/cooperação coparental $(n=10)$, conflito/hostilidade coparental $(n=7)$ e comunicação $(n=2)$. Outras dimensões verificadas incluem triangulação da relação coparental, exposição ao conflito, sabotagem e maternal gatekeeping. Dos 11 estudos, sete mensuraram a coparentalidade por meio de pelo menos duas dimensões. Três estudos utilizaram apenas dimensões positivas da coparentalidade (apoio, comunicação ou cooperação), e um mensurou somente a dimensão de conflito.

Com relação à investigação do comportamento da criança, três estudos utilizaram uma ou mais subescalas do Child Behavior Checklist for Ages 6-18 (CBCL/6-18), versão para crianças e adolescentes entre 6 e 18 anos, para verificar problemas de comportamento infantil (Choi \& Becher, 2019; Herrero et al., 2020; Karberg \& Cabrera, 2020). O Strengths and Difficulties Questionnaire (SDQ), versão para crianças e adolescentes entre 4 e 16 anos, foi utilizado no estudo de Lau (2017) em conjunto com uma escala de autoestima. No estudo de Lamela et al. (2015), utilizaram-se somente as quatro subescalas de dificuldades da criança. Além disso, a subescala de problemas emocionais do SDQ foi respondida por mães e professores no estudo de Baxter et al. (2011), com uma série de perguntas sobre o estado emocional das crianças, conforme seu próprio relato. Outros instrumentos apareceram somente uma única vez nos demais estudos, como o Behavior Problem Index (Dyer et al., 2018), uma versão reduzida do Social Skills Rating System - SSRS (Beckmeyer et al., 2014) e escalas sobre comportamentos resilientes (Pandya, 2017). Em dois estudos, o comportamento da criança foi avaliado por meio de questionário desenvolvido especificamente para eles (Amato et al., 2011; Jiménez-García et al., 2019).

Em relação à qualidade, seis dos dez estudos correlacionais apresentaram alta qualidade metodológica, contemplando sete ou oito itens do checklist (cinco receberam "sim" em todos os itens, e um recebeu somente um "não"). Todos os estudos contemplaram os itens 1 e 2, sobre descrição dos critérios de inclusão da amostra, sujeitos e contexto do estudo. O item menos pontuado foi o 3, sobre a validade do instrumento para avaliação da coparentalidade. Nesse item, cinco estudos não pontuaram. Desses, dois também não pontuaram no item 7, sobre a validade do instrumento para aferição do comportamento infantil (Amato et al., 2011; Jiménez-Garcia et al., 2019), apresentando menor qualidade metodológica.

No estudo de Amato et al. (2011), também não foram identificados todos os dados resultantes de suas análises estatísticas. Por sua vez, o único estudo que não contemplou o item 4, sobre a medida de condição (divórcio/separação), foi o de Baxter et al. (2011), que 
incluiu automaticamente no grupo de mães separadas aquelas que não residiam com a dupla coparental. O único estudo quase experimental (Pandya, 2017) revelou como pontos fracos em termos metodológicos a ausência de estudo de follow-up, a falta de informações sobre o perfil dos participantes incluídos no grupo controle e se estavam recebendo alguma intervenção similar, além da intervenção de interesse (os protocolos utilizados para avaliação da qualidade metodológica podem ser disponibilizados pelo autor correspondente).

\section{Relação entre coparentalidade e comportamento da criança}

Três estudos identificaram diferentes perfis (clusters) de coparentalidade pós-divórcio entre os participantes de acordo com características de relacionamento entre o casal desfeito, associando esses perfis a desfechos de comportamento dos filhos (Tabela 1). Amato et al. (2011) encontraram diferenças significativas nos problemas de comportamento da criança entre os três perfis identificados, sendo menores entre as famílias com coparentalidade cooperativa. Destaca-se que os pais no grupo de coparentalidade cooperativa tinham renda e escolaridade significativamente maiores, enquanto os de menor renda e escolaridade estavam no grupo de parentalidade solo.

Beckmeyer et al. (2014) também encontraram três perfis de coparentalidade, semeIhantes aos de Amato et al. (2011). Contudo, os problemas de comportamento e as habilidades sociais das crianças foram semelhantes em todos os grupos, sem diferenças significativas, o que pode ser explicado pelo fato de todos os participantes da amostra terem sido previamente submetidos a um programa educativo pós-divórcio. Já Lamela et al. (2015) identificaram três diferentes perfis de coparentalidade, denominando-os de coparentalidade de alto conflito, coparentalidade sabotadora e coparentalidade cooperativa. Menos problemas de comportamentos externalizantes foram encontrados no grupo cooperativo em relação aos outros dois grupos. Menos problemas de comportamentos internalizantes foram encontrados entre os pais que exerciam coparentalidade cooperativa em comparação ao grupo de coparentalidade sabotadora, mas não em comparação ao grupo de alto conflito.

Dados de famílias separadas foram comparados aos de famílias casadas e recasadas no estudo longitudinal de Baxter et al. (2011). Os autores mediram somente a existência de hostilidade coparental, identificando-a como um importante fator explicativo do bem-estar emocional dos filhos. A coparentalidade hostil se associou a menor bem-estar emocional em crianças, independentemente do tipo de família (casada, separada ou recasada), tanto na percepção das mães quanto dos filhos. Contudo, entre os pais que tinham uma relação hostil, aqueles que eram separados tinham filhos com piores escores de bem-estar emocional, de acordo com o relato das professoras e das crianças, mas não das mães.

As informações fornecidas pelos pais e pelas crianças também foram diferentes no estudo de Lau (2017), que investigou relações entre as dimensões de apoio, conflito, triangu- 
lação e comunicação coparental. A prática de triangulação na relação coparental não exerceu nenhum impacto significativo no bem-estar dos filhos de acordo com o relato dos pais, mas, de acordo com as respostas fornecidas pelas crianças, sofrer triangulação no conflito coparental representou um importante fator de risco para sua autoestima. Além disso, mais comunicação entre os pais foi associada a mais sintomas emocionais da criança, tanto na percepção dos pais quanto das crianças. Nas análises de regressão, o apoio coparental foi preditor de menos hiperatividade e sintomas emocionais nas crianças, enquanto o conflito coparental foi preditor de maiores escores nesses indicadores.

Lau (2017) realizou uma análise de caminho na qual identificou o efeito indireto do apoio coparental sobre o aumento da hiperatividade da criança por meio do conflito coparental. Além disso, também verificou um efeito indireto da comunicação e do conflito coparental sobre os sintomas emocionais negativos da criança por meio do apoio coparental, além de identificar que havia mais apoio coparental quando a criança era menina. Para explicar esses dados inesperados, verificou que, em relação ao apoio, quem mais o fornecia eram os pais residentes com a criança e hipotetizou que é possível que o conflito decorra de maior sobrecarga de um dos lados, que, além de assumir mais cuidados diretos com a criança por residir com ela, ainda fornece mais apoio do que recebe.

Choi e Becher (2019) identificaram que a idade mais alta da mãe é fator de proteção à coparentalidade, enquanto a depressão materna é fator de risco. No seu modelo explicativo, a coparentalidade positiva foi capaz de predizer menores problemas de comportamento da criança e menor parentalidade severa. Já Karberg e Cabrera (2020) desenvolveram um modelo que explicou $28 \%$ da variação na agressividade infantil, enfatizando uma relação significativa entre instabilidade conjugal e agressividade dos filhos por meio de menor coparentalidade, que era ainda maior para mães divorciadas do que para mães separadas (que nunca chegaram a se casar com suas duplas coparentais) que estavam coabitando com outro parceiro.

No único estudo em que a coparentalidade positiva foi utilizada como moderadora, uma relação entre fenômenos familiares e desfechos da criança (sintomas ansiosos/depressivos e agressividade) foi investigada. Herrero et al. (2020) identificaram que a coparentalidade é um fator protetivo para o comportamento infantil desde que os pais não enfrentem altos níveis de conflito. Assim, demonstraram que o apoio coparental leva a maiores desfechos negativos para crianças cujos pais se encontram em um divórcio de alto conflito, uma vez que pode promover a exposição da criança a um sistema familiar adverso e hostil. Ainda, as autoras identificaram que maior comunicação familiar não se associou a menor conflito coparental, o que sugere que outros fatores fazem com que o conflito se sustente apesar de uma boa comunicação. Tais dados devem ser interpretados com cautela, pois a maioria dos participantes enfrentou um divórcio litigioso à época da coleta. 
No estudo ex post facto conduzido por Jiménez-García et al. (2019) com 317 processos judiciais, foi encontrada maior proporção de problemas externalizantes de comportamento, sintomas emocionais e problemas de desempenho acadêmico entre os filhos de pais que não estavam exercendo a coparentalidade após o divórcio. A análise de regressão logística realizada pelas autoras identificou que a alta intensidade de conflitos sobre a custódia e questões financeiras entre os pais elevou em oito vezes os desfechos negativos na criança, sendo o maior preditor. Mosmann et al. (2018) investigaram crianças e adolescentes com e sem sintomas psicológicos clínicos e identificaram as variáveis competição coparental, prática parental de intrusividade, exposição do filho ao conflito coparental e conflito conjugal como discriminantes dos filhos com sintomas clínicos. Os resultados também mostram as variáveis da coparentalidade com maior poder de discriminação entre os grupos, seguidas das variáveis da parentalidade e da conjugalidade. Dessa forma, compreende-se que a coparentalidade atua como um importante fator interveniente entre a conjugalidade e a parentalidade, pois reflete tanto na relação dos pais com a criança como dos cônjuges.

Já na análise de regressão realizada por Dyer et al. (2018) com dados de 542 pais (homens) de baixa renda, entre os três fatores coparentais analisados (aliança, sabotagem e mediação materna), somente a sabotagem se relacionou positivamente com problemas de comportamento da criança. Por fim, o único estudo experimental retido nesta revisão investigou o efeito dos arranjos coparentais sobre os efeitos de uma intervenção para promoção da espiritualidade entre crianças de famílias divorciadas com alta renda. Desfechos mais positivos foram encontrados em crianças que viviam com pais solteiros ou em arranjos de coparentalidade cooperativa do que em crianças cujos pais tinham arranjos de coparentalidade frequentemente conflitantes ou eram recasados (Pandya, 2017). Detalhes sobre as amostras e os principais resultados dos estudos são apresentados na Tabela 1. 


\section{Tabela 1}

Sistematização dos resultados dos estudos incluídos na revisão sistemática

\begin{tabular}{|c|c|c|}
\hline Autores (ano) & Amostra & Principais resultados \\
\hline $\begin{array}{l}\text { Amato et al. } \\
(2011)\end{array}$ & $\begin{array}{l}\text { Participaram } 944 \text { duplas de pais/filhos; filhos com idade entre } 7 \text { e } 19 \\
\text { anos. Os pais no grupo de coparentalidade cooperativa tinham renda e } \\
\text { escolaridade significativamente maiores, enquanto os de menor renda e } \\
\text { escolaridade estavam no grupo de parentalidade solo. O grupo de } \\
\text { coparentalidade cooperativa estava divorciado. }\end{array}$ & $\begin{array}{l}\text { Identificação de três diferentes perfis de coparentalidade após o divórcio } \\
\text { dos pais: coparentalidade cooperativa (percepção de altos níveis de } \\
\text { envolvimento, comunicação e cooperação coparental com o pai não } \\
\text { residente); coparentalidade paralela (pais não residentes com a criança } \\
\text { têm envolvimento na vida dos filhos, mas se comunicam pouco com os } \\
\text { pais residentes e são percebidos por estes como tendo uma participação } \\
\text { limitada na vida das crianças); e parentalidade solo (pais não residentes } \\
\text { pouco viam a criança). Os problemas de comportamento da criança } \\
\text { foram significativamente menores no grupo de coparentalidade } \\
\text { cooperativa. }\end{array}$ \\
\hline $\begin{array}{l}\text { Baxter et al. } \\
(2011)\end{array}$ & $\begin{array}{l}\text { Participaram } 4.303 \text { crianças, das quais } 3.640 \text { ( } 85 \% \text { ) viviam com ambos } \\
\text { os pais biológicos; } 663 \text { ( } 15 \% \text { ), com a mãe e o padrasto; e } 560 \text { ( } 13 \% \text { ) } \\
\text { viviam somente com a mãe. Dados coletados entre } 2004 \text { e } 2006 \text {. } \\
\text { Respostas das mães foram coletadas para } 4.189 \text { crianças. Respostas } \\
\text { de professores foram coletadas para } 3.487 \text { crianças. Com relação às } \\
\text { crianças, } 4.220 \text { forneceram respostas na segunda etapa da coleta } \\
\text { (a criança tinha entre } 4 \text { e } 5 \text { anos na primeira etapa e entre } 6 \text { e } 7 \text { anos } \\
\text { na segunda etapa). }\end{array}$ & $\begin{array}{l}\text { Crianças com 6-7 anos que vivem com os pais biológicos têm melhor } \\
\text { bem-estar emocional do que aquelas que vivem somente com a mãe, } \\
\text { sendo a hostilidade coparental significativamente associada (e um } \\
\text { importante fator explicativo) ao bem-estar emocional das crianças. } \\
\text { Especificamente, independentemente do tipo de família (casada ou } \\
\text { separada), crianças cujos pais tinham uma relação coparental hostil } \\
\text { tinham pior bem-estar emocional do que aquelas cujos pais não } \\
\text { demonstravam hostilidade. Entre os pais que tinham uma relação hostil, } \\
\text { aqueles que eram separados tinham filhos com piores escores em } \\
\text { bem-estar emocional, de acordo com o relato das professoras e das } \\
\text { crianças, mas não das mães. }\end{array}$ \\
\hline $\begin{array}{l}\text { Beckmeyer et al. } \\
(2014)\end{array}$ & $\begin{array}{l}\text { Participaram do estudo } 270 \text { pessoas. A maioria dos participantes era } \\
\text { composta de mães e brancas e com algum grau de ensino superior. } \\
\text { Todos os participantes já haviam passado por um programa educativo } \\
\text { pós-divórcio. }\end{array}$ & $\begin{array}{l}\text { Identificação de três perfis de coparentalidade pós-divórcio, } \\
\text { nomeadamente: coparentalidade cooperativa e envolvida; } \\
\text { coparentalidade moderada; e coparentalidade infrequente e conflitosa. }\end{array}$ \\
\hline
\end{tabular}




\begin{tabular}{ll}
\multicolumn{1}{c}{ Autores (ano) } & \multicolumn{1}{c}{ Amostra } \\
\hline Lamela et al. & Participaram 314 pais: 71,3\% de mulheres, média de 5,19 anos de \\
$(2015)$. & divórcio e alto nível de escolaridade.
\end{tabular}

(2015). divórcio e alto nível de escolaridade.

Lau (2017)

Participaram 142 pais separados, sendo 115 mulheres. Somente oito participantes eram recasadas. A média de idade das crianças foi 10 anos, com $47,2 \%$ tendo entre 12 e 17 anos.

Pandya (2017)

Participaram 1.893 crianças de 9 a 12 anos de classe média alta e elite, de 15 diferentes países. Medidas extraídas a partir do relato das crianças.

Dyer et al. (2018) Participaram 542 pais (somente homens) que não residiam com a criança-focal e tinham baixa renda.
Principais resultados

Identificação de três perfis de coparentalidade: coparentalidade de alto conflito (pouca concordância, menor escore de todos em divisão das tarefas, alta sabotagem e conflito); coparentalidade sabotadora (escores altos em sabotagem e baixo/médio em concordância, divisão de tarefas e conflito); coparentalidade cooperativa (escores altos em concordância, divisão de tarefas, escores baixos em sabotagem e conflitos). O grupo da coparentalidade cooperativa reportou significativamente menos problemas de comportamentos internalizantes nos filhos do que no grupo de coparentalidade sabotadora. O grupo de coparentalidade cooperativa relatou escores significativamente menores em problemas de comportamentos externalizantes do que os outros grupos.

Análises de regressão diferentes foram conduzidas com dados fornecidos pelos pais e pelas crianças. De acordo com os pais, o apoio coparental predisse menores sintomas de hiperatividade e sintomas emocionais. $\mathrm{O}$ conflito coparental predisse maiores sintomas de hiperatividade e sintomas emocionais. Mais comunicação entre os pais foi associada a mais sintomas emocionais entre as crianças.

Crianças que viviam com pais solteiros ou em arranjos de coparentalidade cooperativa entre seus pais pontuaram mais alto nas escalas de resiliência e força em relação àquelas que tinham arranjos de coparentalidade frequentemente conflitantes ou viviam com pais recasados.

O estudo de desenvolvimento e validação da escala de coparentalidade identificou três fatores: aliança, sabotagem e maternal gatekeeping (mediação materna). Nas análises de correlação, sabotagem se relacionou moderada e significativamente com problemas de comportamento da criança. Na análise de validação preditiva (regressão múltipla), somente sabotagem se relacionou positiva e

significativamente com problemas de comportamento da criança. 


\begin{tabular}{ll}
\multicolumn{1}{c}{ Autores (ano) } & \multicolumn{1}{c}{ Amostra } \\
\hline Jiménez-García & Estudo ex post facto com 317 processos judiciais de separação/divórcio \\
et al. (2019) & correspondentes aos anos de 2009 a 2016.
\end{tabular}

$\begin{array}{ll}\text { Jiménez-García } & \text { Estudo ex post facto com } 317 \text { processos judiciai } \\ \text { et al. (2019) } & \text { correspondentes aos anos de } 2009 \text { a } 2016 .\end{array}$

Choi e

Becher (2019)

Karberg e

Cabrera (2020)

Herrero et al.

(2020)
Participaram 1.773 mães separadas. Dados coletados entre 1998 e 2000 - estudo longitudinal. A maioria das participantes era relativamente jovem (média de 26 anos de idade quando a criança tinha aproximadamente 3 anos) e negras $(64,2 \%)$ - 44,7\% não tinham nenhum contato com o pai da criança.

Participaram 3.387 crianças que residiam com as mães biológicas. A coparentalidade foi medida quando a criança tinha 5 anos. A agressividade foi medida quando a criança tinha 9 anos. Dados coletados entre 1998 e 2000. Amostra representativa para população de grandes cidades dos Estados Unidos.

Participaram 309 pais (39\%) e mães (61\%) divorciados - $71 \%$ tinham a custódia da criança. A maioria (79\%) estava em divórcio litigioso com $62 \%$ em disputa administrativa/judicial $-45 \%$ referiram ter uma relação "não existente" com a dupla coparental.

\section{Principais resultados}

Houve maior proporção de tristeza, agressividade, desobediência, baixa autoestima, comportamentos autolesivos, ansiedade e irritação, e decréscimo do desempenho acadêmico das crianças entre aqueles pais que não exercitavam a coparentalidade. Nas análises de regressão logística, o exercício da coparentalidade não foi incluído. O preditor mais forte foi a alta intensidade de conflito, indicando que, quando os pais apresentavam alta intensidade de conflito, aumentavam em oito vezes os desfechos negativos na criança.

A coparentalidade de apoio foi utilizada como mediadora entre dados sociodemográficos e sintomas depressivos maternos e parentalidade severa e problemas de comportamento infantil, em uma análise de modelagem de equações estruturais. Mães mais novas apresentaram menor coparentalidade positiva e maior parentalidade severa. Mais sintomas depressivos da mãe predisseram menor coparentalidade positiva, e maior nível de escolaridade dos pais foi capaz de predizer maior coparentalidade positiva. A coparentalidade positiva foi capaz de predizer menos problemas de comportamento da criança e menor parentalidade severa.

Uma análise de caminhos foi conduzida, sendo a variável independente a instabilidade conjugal, e a variável dependente, o comportamento agressivo da criança. Coparentalidade positiva, envolvimento paterno, envolvimento materno e responsividade materna foram incluídos como variáveis mediadoras. O estado civil (casada ou não) foi utilizado como moderador entre a variável independente e as mediadoras. A associação entre instabilidade conjugal e agressividade infantil por meio do apoio coparental foi mais forte para mães divorciadas do que para mães separadas do pai da criança que estavam com outro parceiro.

A coparentalidade positiva foi utilizada como moderadora em uma análise de caminhos, moderando a relação entre fenômenos familiares (comunicação familiar, dificuldades psicológicas dos pais, conflito com a dupla coparental e consequências socioeconômicas para as crianças) e sintomas ansiosos/depressivos e agressividade da criança. Maior comunicação familiar se relacionou a níveis mais baixos de sintomas ansiosos/depressivos e agressividade. 


\section{Discussão}

A presente revisão sistemática teve como objetivo analisar as relações entre coparentalidade e o comportamento da criança em famílias divorciadas com base em estudos conduzidos nos últimos dez anos. Partiu-se do pressuposto de que a coparentalidade é um fator relevante para desfechos no desenvolvimento infantil, especialmente após a separação ou o divórcio, evento estressor que implica modificações na estrutura e dinâmica familiar, conforme indicado por McGoldrick e Shibusawa (2016). Contudo, a qualidade da coparentalidade é diferente para cada integrante da dupla coparental e, ainda, percebida de maneira específica por cada criança (McHale \& Sirotkin, 2019), sendo importante destacar as características amostrais dos 11 estudos incluídos na revisão.

A predominância de mães nas amostras tem sido identificada em outras revisões de literatura sobre fenômenos do contexto familiar (Coltro et al., 2020; Samda et al., 2020), apesar de mudanças sociais apontarem para uma progressivamente maior participação paterna no contexto doméstico e nos cuidados com os filhos (Gomes \& Alvarenga, 2016). Além disso, destaca-se que nenhum estudo investigou a percepção de ambos os integrantes da dupla coparental sobre a coparentalidade ou sobre os comportamentos da criança, o que representa uma limitação metodológica, já que as impressões sobre a dinâmica familiar permanecem parciais. No que diz respeito a famílias divorciadas, pode ser ainda mais importante incluir ambos os pais na amostra, o que contribui para a identificação de percepções diferentes sobre a coparentalidade e o comportamento dos filhos com base no tempo de convivência com a criança.

Entre os 11 estudos aqui analisados, oito incluíram alguma medida sobre conflito, hostilidade ou sabotagem entre os pais, gerando resultados consistentes sobre os efeitos negativos de uma relação coparental conflituosa para o comportamento das crianças. Teubert e Pinquart (2010) conduziram uma metanálise em que também identificaram uma relação positiva entre conflito coparental e comportamentos externalizantes dos filhos, enquanto a triangulação e a baixa cooperação coparental estiveram mais associadas a comportamentos internalizantes. Essa diferença não foi identificada no estudo de Lamela et al. (2015), que encontrou níveis semelhantes de comportamentos internalizantes e externalizantes entre os grupos de coparentalidade sabotadora e coparentalidade de alto conflito. Mosmann et al. (2017) encontraram, em sua pesquisa com amostra brasileira, um poder preditivo das variáveis da coparentalidade sobre os sintomas internalizantes e externalizantes dos filhos, o qual era preponderante nas dimensões da conjugalidade e da parentalidade, corroborando achados de outros países (Feinberg, 2003; Teubert \& Pinquart, 2010). De forma semelhante, Lau (2017) identificou uma relação positiva entre conflito coparental e hiperatividade e sintomas emocionais, mas não sobre problemas de conduta. Contudo, identificou que a triangulação coparental atingiu negativa e significativamente a autoestima das crianças somente conforme o autorre- 
lato delas, não na percepção das mães (Lau, 2017), convergindo com a análise de Teubert e Pinquart (2010).

Os achados desta revisão confirmam os de Lamela e Figueiredo (2016) e Raposo et al. (2011), sustentando o conflito pós-divórcio entre os pais como o mais prejudicial para os filhos, mesmo que o conflito não esteja necessariamente relacionado à dimensão coparental. Ainda que Baxter et al. (2011) tenham referido a medida de conflito como referente a conflito coparental, uma análise detalhada dos itens utilizados para construir esse indicador revelou que o conflito medido não se referia apenas às decisões sobre a criança. Da mesma forma, os diferentes tipos de intensidade de conflito investigados por Jiménez-García et al. (2019) revelam que, no contexto do divórcio litigioso, disputas a respeito de aspectos financeiros e sobre a custódia dos filhos foram as maiores preditoras de desfechos negativos na criança.

Constatou-se que o apoio coparental contribuiu significativamente para melhores níveis de ajustamento psicológico das crianças, reverberando positivamente nas relações familiares. Souza (2018) investigou as repercussões da coparentalidade e do envolvimento paterno no comportamento da criança no Brasil, e os resultados apontaram a importância do relacionamento coparental positivo e do envolvimento paterno (cuidados básicos e brincadeiras) para a compreensão dos comportamentos dos pré-escolares, principalmente quando se consideram seus impactos adaptativos e desadaptativos para o desenvolvimento infantil. Contudo, considerando as interações complexas entre as dimensões coparentais, parece ser relevante identificar como e por quem o apoio coparental é fornecido. As análises apresentadas por Lau (2017), por exemplo, evidenciam que o apoio coparental fornecido pelo cuidador que reside com a criança em comparação ao cuidador que não reside com ela pode indicar a presença de sobrecarga unilateral dos cuidados com a criança, o que tende a gerar desfechos negativos sobre o comportamento dos filhos.

Portanto, com vistas a melhor compreender os impactos do apoio coparental sobre os filhos, é preciso incluir nas análises fatores de caracterização dos pais e dos filhos, bem como do contexto em que as famílias se encontram. Em situações de maior condição socioeconômica e escolaridade, por exemplo, é esperado que os pais sejam capazes de oferecer maior apoio coparental, como relatado por Amato et al. (2011).

Com relação à comunicação coparental, os estudos apresentaram efeitos contrários ao esperado (Herrero et al., 2020; Lau, 2017). Assim, o simples fato de ter mais comunicação entre os pais não é necessariamente positivo para as crianças nem ajuda a promover seu desenvolvimento, especialmente se essa comunicação é acompanhada por níveis mais altos de conflito coparental. Feinberg (2003) afirma que é papel dos pais controlar o próprio comportamento, cuidando da forma com que se comunicam, um com o outro, evitando discutir temas conjugais, brigar e/ou alterar a voz na frente da criança. Segundo ele, os pais devem esperar 
um momento no qual a criança não esteja presente, para que possam resolver esse tipo de questão.

Além disso, o autor destaca que nem todos os conflitos são prejudiciais à criança. Nos casos em que os pais conseguem manejar os conflitos de forma positiva, os impactos para os filhos podem ser benéficos (ou, pelo menos, não prejudiciais), na medida em que a criança observa modelos de como resolver conflitos de maneira desejável, podendo aprender com o exemplo dos pais. Segrin (2006) demonstrou que famílias que recorrem a estratégias positivas de comunicação e que procuram gerar interações harmoniosas entre os seus membros tendem a ser mais saudáveis do que aquelas que mantêm relações conflituosas.

O divórcio nem sempre é um evento negativo, sendo, em muitos casos, a melhor solução quando os conflitos entre o casal passam a ser rotineiros. A qualidade da coparentalidade pode impactar de forma positiva o comportamento dos filhos após a separação/divórcio. Souza (2000) investigou como os filhos vivenciam a separação dos pais e concluiu que as crianças têm sua saúde mental associada ao bem-estar dos pais e à qualidade do relacionamento estabelecido entre ambos. As crianças correm riscos, portanto, quando crescem em uma família em que o casal está em constante conflito, quer os pais vivam juntos ou separados. A autora acrescenta que os filhos de pais divorciados poderão ser competentes e bem ajustados quando o divórcio puder conter a demanda de conflitos entre os cônjuges e quando a mãe ou o pai que tiver a guarda da criança for capaz de proporcionar um ambiente de cuidado positivo, a despeito do estresse associado a um papel singular mais sobrecarregado.

Quando a separação do casal é inevitável, é necessário que os conflitos gerados a partir de tal escolha possam representar lições de negociação e de entendimento para o desenvolvimento das crianças. A criação satisfatória e o desenvolvimento saudável de uma criança envolvem um conjunto de cuidados físicos, cognitivos, emocionais, afetivos e instrutivos pelos quais ambos os pais são responsáveis, independentemente de estarem casados ou separados (Goetz \& Vieira, 2010). Dessa forma, a qualidade da coparentalidade e da comunicação, o apoio coparental e o baixo conflito são alguns dos elementos importantes quando as famílias passam por um processo de separação/divórcio.

\section{Considerações finais}

A coparentalidade é um fenômeno de central importância para a compreensão das relações entre pais e filhos e dos impactos do exercício compartilhado de cuidado com a criança sobre seu desenvolvimento. No contexto do divórcio, a coparentalidade é um dos subsistemas mais vulneráveis às modificações da estrutura familiar, requerendo a definição de novos acordos e compromissos dos pais para/com seus filhos e ex-cônjuges. A maioria dos estudos retidos nesta revisão sistemática apresentaram qualidade metodológica suficiente para que seus dados contribuam para o acumulado de evidências empíricas a respeito das relações entre 
coparentalidade e comportamento infantil em famílias divorciadas. Seus resultados contribuem para a compreensão da coparentalidade como fenômeno multidimensional, em que diferentes padrões de composição a partir de suas dimensões tendem a gerar resultados distintos sobre o comportamento infantil. Portanto, uma análise mais complexa da coparentalidade é contemplada quando ao menos duas ou mais dimensões são consideradas, bem como suas interações.

Ademais, os resultados e as discussões apresentados demonstram que, embora o apoio coparental seja benéfico para desfechos positivos no comportamento da criança, é preciso levar em conta as características da família e do contexto familiar para melhor compreender essas relações. Da mesma maneira, o conflito parental permanece sendo indicado como fator de risco ao desenvolvimento dos filhos, relacionando-se com comportamentos externalizantes e/ou internalizantes. Contudo, esse resultado não é unânime, variando de acordo com o contexto do divórcio. Dessa forma, sugere-se que a coparentalidade é multideterminada por fatores diversos, como defende o Modelo Ecológico da Coparentalidade proposto por Feinberg (2003).

Portanto, esta revisão se soma aos demais estudos científicos sobre coparentalidade ao fornecer uma análise rigorosa dos aspectos metodológicos e dos resultados dos artigos retidos. Destacam-se como contribuições relevantes a verificação de interações complexas das dimensões da coparentalidade não somente com elementos contextuais, mas também entre si para produzir efeitos sobre o comportamento da criança, e o apontamento do divórcio como contexto peculiar de transformação da relação coparental a partir da reconfiguração da estrutura familiar.

Analisando a qualidade da evidência, destacamos como limitação dos estudos a fragilidade das medidas utilizadas para aferir as dimensões da coparentalidade, além de cinco trabalhos não terem indicado a validade psicométrica deles (Amato et al., 2011; Baxter et al., 2011; Choi \& Becker, 2019; Jiménez-García et al., 2018; Karberg et al., 2020). Tal fato prejudica a generalização dos resultados e reduz a possibilidade de replicação dos desenhos de pesquisa utilizados. Além disso, a predominância de mães como participantes indica um viés amostral relevante. Assim, recomenda-se que estudos futuros investiguem a coparentalidade a partir da perspectiva de homens (pais) e também da criança, e invistam na coleta de dados de ambos os integrantes da dupla coparental, o que fornecerá um quadro mais fidedigno das relações familiares. Além disso, recomenda-se o uso de instrumentos e medidas validados para a população estudada e que contemplem um maior número de dimensões da parentalidade. No contexto do divórcio, sobretudo, parece importante inserir como variáveis de controle nas análises o tempo desde o divórcio e outros fatores familiares e contextuais.

A presente revisão sistemática apresenta como limitações metodológicas a exclusão de materiais de literatura cinzenta e a não realização da etapa de consulta com especialistas para 
reduzir possíveis perdas de materiais relevantes. Apesar disso, cabe destaque à etapa de consulta na lista de referências dos estudos incluídos e o uso do Guia PRISMA e de protocolos padronizados para avaliação da qualidade metodológica dos estudos incluídos. Outra limitação foi o fato de não termos registrado o protocolo de investigação, mas ele fica disponível mediante solicitação ao autor correspondente.

Em suma, esta revisão sistemática fornece dados consistentes que podem fundamentar a prática profissional de psicólogos envolvidos com a avaliação psicossocial dos sistemas familiares no contexto divórcio e na intervenção com famílias e crianças para a promoção do desenvolvimento infantil. Para os profissionais envolvidos com famílias em processo de divórcio, especificamente nos contextos clínico, jurídico e de assistência social, esta revisão fornece subsídios para o atendimento das famílias com vistas à proteção da infância e para as tomadas de decisão baseadas em evidências. Sobretudo, destaca-se a relevância científica e social do investimento em pesquisas que contribuam para a análise do complexo interjogo entre os fatores individuais, familiares e contextuais no cenário brasileiro. 


\section{Referências}

Amato, P. R., Kane, J. B., \& James, S. (2011). Reconsidering the "Good Divorce". Family Relations, 60(5), 511-524. https://doi.org/10.1111/j.1741-3729.2011.00666.x

Baxter, J., Weston, R., \& Qu, L. (2011). Family Structure, co-parental relationship quality, post-separation paternal involvement, and children's emotional Wellbeing. Journal of Family Studies, 17(2), 86-109. https://doi.org/10.5172/jfs.2011.17.2.86

Beckmeyer, J. J., Coleman, M., \& Ganong, L. H. (2014). Postdivorce Coparenting Typologies and Children's Adjustment. Family Relations, 63(4), 526-537. https://doi.org/10.1111/fare.12086

Bolsoni-Silva, A. T., Loureiro, S. R., \& Marturano, E. M. (2016). Comportamentos internalizantes: Sociais, associações com habilidades práticas educativas, recursos do ambiente familiar e depressão materna. Psico, 47(2), 111-120. https://doi.org/10.15448/1980-8623.2016.2.20806

Choi, J. K., \& Becher, E. H. (2019). Supportive Coparenting, Parenting Stress, Harsh Parenting, and Child Behavior Problems in Nonmarital Families. Family Process, 58(2), 404-417. https://doi. org/10.1111/famp.12373

Coltro, B. P., Paraventi, L., \& Vieira, M. L., (2020). Relações entre Parentalidade e Apoio Social: Revisão integrativa de literatura. Contextos Clínicos, 13(1), 1-26. https://doi.org/10.4013/ ctc.2020.131.12

Cusí, L. M., Günther-Bel, C., Puigdesens, A. V., Orriols, M. C., \& Costa, J. L. M. (2020). Instruments for the Assessment of Coparenting: A systematic review. Journal of Child and Family Studies, 29(9), 2487-2506. https://doi.org/10.1007/s10826-020-01769-3

Dyer, W. J., Fagan, J., Kaufman, R., Pearson, J., \& Cabrera, N. (2018). Self-perceived Coparenting of Nonresident Fathers: Scale Development and Validation. Family Process, 57(4), 927-946. https://doi.org/10.1111/famp.12331

Feinberg, M. E. (2003). The Internal Structure and Ecological Context of Coparenting: A Framework for Research and Intervention. Parenting: Science and Practice, 3(2), 95-131. https://doi. org/10.1207/S15327922PAR0302_01

Goetz, E., \& Vieira, M. L. (2010). Pai real, pai ideal: O papel paterno no desenvolvimento infantil. Juruá.

Gomes, Q. S., \& Alvarenga, P. (2016). O envolvimento paterno em famílias de diferentes níveis socioeconômicos. Psicologia: Teoria e Pesquisa, 32(3), 1-9. https://doi.org/10.1590/ 0102-3772e323216

Greene, S. M., Anderson, E. R., Forgatch, M. S., DeGarmo, D. S., \& Hetherington, E. M. (2016). Risco e resiliência após o divórico. In F. Walsh (Ed.), Processos Normativos da Família: Diversidade e Complexidade (4th ed., pp. 102-127). Artmed.

Hadfield, K., Amos, M., Ungar, M., Gosselin, J., \& Ganong, L. (2018). Do Changes to Family Structure Affect Child and Family Outcomes? A Systematic Review of the Instability Hypothesis. Journal of Family Theory and Review, 10(1), 87-110. https://doi.org/10.1111/jftr.12243

Herrero, M., Martínez-Pampliega, A., \& Alvarez, I. (2020). Family Communication, Adaptation to Divorce and Children's Maladjustment: The Moderating Role of Coparenting. Journal of Family Communication, 20(2), 114-128. https://doi.org/10.1080/15267431.2020.1723592 
Instituto Brasileiro de Geografia e Estatística [IBGE]. (2019). Estatísticas do registro civil 2018. https:// biblioteca.ibge.gov.br/visualizacao/periodicos/135/rc_2018_v45_informativo.pdf

Jiménez-García, P., Contreras, L., \& Cano- Lozano, M. C. (2019, January). Types and intensity of postdivorce conflicts, the exercise of coparenting and its consequences on children. Revista Iberoamericana de Psicología Y Salud, 10(1), 48-63. https://doi.org/10.23923/j.rips.2019.01.025

Joanna Briggs Institute. (2020). Critical Appraisal Checklist for Quasi-Experimental Studies. Joanna Briggs Institute.

Karberg, E., \& Cabrera, N., J. (2020). Children's Adjustment Parents' Breakup: The Mediational Effects of Parenting and Coparenting. Journal of Family Issues, 41(10), 1810-1833. https://doi. org/10.1177\%2F0192513X19894347

Lau, Y. K. (2017). Postdivorce Coparenting and Children's Well-Being in Hong Kong. Journal of Divorce \& Remarriage, 58(5), 329-346. https://doi.org/10.1080/10502556.2017.1302237

Lamela, D., Figueiredo, B., Bastos, A., \& Feinberg, M. (2015). Typologies of Post-divorce Coparenting and Parental Well-Being, Parenting Quality and Children's Psychological Adjustment. Child Psychiatry \& Human Development, 47, 823-1006. https://doi.org/10.1007/s10578-015-0604-5

Lamela, D., \& Figueiredo, B. (2016). Coparenting after marital dissolution and children's mental health: A systematic review. Jornal de Pediatria, 92(4), 331-342. https://doi.org/10.1016/j. jped.2015.09.011

Margolin, G., Gordis, E. B., \& John, R. S. (2001). Coparenting: A link between marital conflict and parenting in two-parent families. Journal of Family Psychology, 15(1), 3-21. https://doi. org/10.1037/0893-3200.15.1.3

McGoldrick, M., \& Shibusawa, T. (2016). O ciclo vital familiar. In F. Walsh (Ed.), Processos Normativos da Familia: Diversidade e Complexidade (4th ed., pp. 375-398). Artmed.

McHale, J. P., Kuersten-Hogan, R., \& Rao, N. (2004) Growing points for coparenting theory and research. Journal of Adult Development, 11(3), 221-234. https://doi.org/10.1023 /B:JADE.0000035629.29960.ed

McHale, J. P., \& Sirotkin, J. T. (2019). Coparenting in diverse family systems. In M. H. Bornstein (Ed.), Handbook of parenting: Being and becoming a parent (Vol.3, pp. 15-37). Routledge.

Moher, D., Liberati, A., Tetzlaff, J., Altman, D. G., \& Prisma Group. (2009). Preferred reporting items for systematic reviews and meta-analyses: The PRISMA statement. PloS Medicine, 6(7). https:// doi.org/10.1371/journal.pmed.1000097

Mosmann, C. P., Costa, C. B., Einsfeld, P., Silva, A. G. M.., \& Koch, C. (2017). Conjugalidade, parentalidade e coparentalidade: Associações com sintomas externalizantes e internalizantes em crianças e adolescentes. Estudos de psicologia, 34(4). https://doi.org/10.1590 /1982-02752017000400005

Mosmann, C., Costa, C. B., Silva, A. G. M., \& Luz, S. K. (2018). Filhos com sintomas psicológicos clínicos: Papel discriminante da conjugalidade, coparentalidade e parentalidade. Trends in Psychology, 26(1), 429-442. https://doi.org/10.9788/tp2018.1-17pt

Ouzzani, M., Hammady, H., Fedorowicz, Z., \& Elmagarmid, A. (2016). Rayyan - a web and mobile app for systematic reviews. Systematic Reviews, 5(210). https://doi.org/10.1186/s13643-016-0384-4 
Pandya, S. P. (2017). Spirituality for Building Resilience in Children of Divorced Parents: A Cross-Country Experimental Study. Journal of Divorce \& Remarriage, 58(6), 447-468. https://doi.org/ 10.1080/10502556.2017.1343554

Raley, R. K., \& Sweeney, M. M. (2020). Divorce, Repartnering, and Stepfamilies: A Decade in Review. Journal of Marriage and Family, 82(1), 81-99. https://doi.org/10.1111/jomf.12651

Raposo, H. S., Figueiredo, B. F., Lamela, D. J., Nunes-Costa, R. A., Castro, M. C., \& Prego, J. (2011). Ajustamento da criança à separação ou divórcio dos pais. Revista de psiquiatria clínica, 38(1), 29-33. https://doi.org/10.1590/S0101-60832011000100007

Samdan, G., Kiel, N., Petermann, N., Rothenfuber, S., Zierul, C., \& Reinelt, T. (2020). The relationship between parental behavior and infant regulation: A Systematic review. Developmental Review, 57, 100923. https://doi.org/10.1016/j.dr.2020.100923

Sands, A., Thompson, E. J., \& Gaysina, D. (2017). Long-term influences of parental divorce on offspring affective disorders: A systematic review and meta-analysis. Journal of Affective Disorders, 218, 105-114. https://doi.org/10.1016/j.jad.2017.04.015

Segrin, C. (2006). Invited article: Family interactions and well-being: Integrative perspectives. Journal of Family Communication, 6, 3-21. https://doi.org/10.1207/s15327698jfc0601_2

Solmeyer, A. R., Feinberg, M. E., Coffman, D. L., \& Jones, D. E. (2014). The Effects of the Family Foundations Prevention Program on Coparenting and Child Adjustment: A Mediation Analysis. Prevention Science, 15(2), 213-223. https://doi.org/10.1007/s11121-013-0366-x

Souza, R. M. (2000). Depois que papai e mamãe se separaram: Um relato dos filhos. Psicologia: Teoria e Pesquisa, 16(3), 203-211. https://doi.org/10.1590/S0102-37722000000300003

Souza, C. D. (2018). Repercussões da Coparentalidade e do Envolvimento paterno no comportamento da criança pré-escolar em famílias biparentais. [Unpublished Doctoral thesis]. Universidade Federal de Santa Catarina.

Teubert, D., \& Pinquart, M. (2010). The Association Between Coparenting and Child Adjustment: A meta-analysis. Parenting, 10(4), 286-307. https://doi.org/10.1080/15295192.2010.492040

Zemp, M., Johnson, M. D., \& Bodenmann, G. (2018). Within-family processes: Interparental and coparenting conflict and child adjustment. Journal of Family Psychology, 32(3), 299-309. https:// doi.org/10.1037/famooo0368 
EQUIPE EDITORIAL

Editora-chefe

Cristiane Silvestre de Paula

\section{Editores associados}

Alessandra Gotuzo Seabra

Ana Alexandra Caldas Osório

Luiz Renato Rodrigues Carreiro

Maria Cristina Triguero

Veloz Teixeira

\section{Editores de seção}

"Avaliação Psicológica"

Alexandre Serpa

André Luiz de Carvalho

Braule Pinto

Luiz Renato Rodrigues Carreiro

Vera Lúcia Esteves Mateus

"Psicologia e Educação"

Alessandra Gotuzo Seabra

Carlo Schmidt

Regina Basso Zanon
"Psicologia Social e Saúde das

Populações"

Enzo Banti Bissoli

Marina Xavier Carpena

\section{"Psicologia Clínica"}

Carolina Andrea Ziebold Jorquera

Julia Garcia Durand

Natalia Becker

\section{"Desenvolvimento Humano"}

Maria Cristina Triguero

Veloz Teixeira

Rosane Lowenthal

\section{Suporte técnico}

Maria Fernanda Liuti

Bento da Silva

Camila Fragoso Ribeiro
PRODUÇÃO EDITORIAL

Coordenação editorial

Ana Claudia de Mauro

Estagiários editoriais

Júlia Lins Reis

Pietro Menezes

Élcio Carvalho

Preparação de originais

Carlos Villarruel

\section{Revisão}

Vera Ayres

\section{Diagramação}

Acqua Estúdio Gráfico 